\title{
Surface roughness and refractive index changes in contact lens induced by lens care systems.
}

$\underline{\text { Lira M}}^{1}$, Franco S, Vazquez-Dorrio JB, Real Oliveira ME, Costa MF.

\section{Author information}

${ }^{1}$ Centre of Physics (CFUM) (M.L., M.E.C.D.R.O., M.F.M.C.), University of Minho, Braga, Portugal; and Department of Physics (J.B.V-D.), University of Vigo, Pontevedra, Spain.

\begin{abstract}
PURPOSE:

The aim of this study was to analyze the influence of different lens care systems in surface roughness and refractive index $(\mathrm{Rl})$ of contact lenses $(\mathrm{CL})$. This information provides us with a better understanding of how care solutions affect CL materials.
\end{abstract}

\section{METHODS:}

Several $C L$ and three commercially available and appropriate lens care solutions were used (two polyhexamethylene biguanide and one hydrogen peroxide care systems). Lenses were immersed in the lens care systems, and then measurements with CLR 12-70 digital automated refractometer and atomic force microscopy analysis on Tapping mode were recorded. The measurements were performed before and after the lenses were immersed in each care solution.

\section{RESULTS:}

Significant changes were observed on the CL materials when exposed to lens care systems. All the materials changed, to a greater or lesser extent, their roughness and $\mathrm{RI}$, after being immersed in the different solutions. The water content varied between $0 \%$ (Nelfilcon A in ReNu Multiplus, Senofilcon A in AOSEPT Plus, and Methafilcon A in Solocare Aqua) and 4.1\% (Hilafilcon B in Solocare Aqua) The higher change in roughness was obtained with ReNu Multiplus in the lens Comfilcon A (with an increase of $27.2 \mathrm{~nm}$ ) and Senofilcon A (with an increase of $16.7 \mathrm{~nm}$ ).

\section{CONCLUSION:}

This study suggests that lens care systems play an important role in surface roughness and RI of $\mathrm{CL}$. 